\title{
Thermal Simulations of Packaged IR LED Arrays
}

\author{
John V. Lawler*a and Joseph Currano ${ }^{\mathrm{a}}$ \\ ${ }^{a}$ ATEC, Inc., 7100 Baltimore Avenue, Suite 300, College Park, MD 20790
}

\begin{abstract}
The steady-state and transient behaviors of packaged IR LED arrays have been studied via numerical simulations. The waste heat generated by LEDs must be removed through a cold plate or a cryogenic cold finger attached to the backside of the driver array. Therefore, this heat must travel across the LED array-driver interface and through the driver array. The modeling results demonstrate that the thermal resistance of these components can be significant. The steady-state temperature profiles across several configurations are used to identify the thermal bottlenecks. Transient simulations are used to quantify the rise and fall times of the IR LEDs, and the fall times can be significantly reduced by changes in the LED layout. These proposed guidelines to minimize thermal issues in LED arrays should result in better performing and more reliable IR LED arrays.
\end{abstract}

Keywords: thermal management, light emitting diodes, LED, infra-red, scene generation

\section{INTRODUCTION}

The need for increasingly sensitive infrared (IR) systems for detection of moving objects with temperatures that differ from ambient has led to substantial research on both linear detector arrays and detector matrices in the main atmospheric infrared bands (or windows) from 3 to $5 \mu \mathrm{m}$ and 8 to $14 \mu \mathrm{m}$. The development and manufacturing of these systems also creates demand for light sources that can be used to calibrate and test detectors in these bands, particularly light sources that are capable of simulating temperature distributions representative of conditions found in the field. This need includes large area sources that are capable of simulating temperature distributions up to $700 \mathrm{~K}$, which are typically used to establish the base line of the detector and eliminate distortions of the scene (object view) in the IR. It also includes sources with small lateral feature size used for evaluation of the system resolution.

Current infra-red (IR) projection systems for Hardware-in-the-Loop (HWIL) simulations use resistive element arrays to simulate infrared objects at temperatures up to $700 \mathrm{~K}$. Resistive element arrays have many benefits. It is a mature IR scene projection technology. Resistively heated elements provide acceptable response time, temperature resolution, good spatial resolution, and adequate frame rates. However, future HWIL systems have requirements that exceed the capability of current resistive element arrays. Future HWIL systems will require the simulation of point and spatially extended targets at apparent temperatures in excess of $3000 \mathrm{~K}$.

Resistive element arrays have several inherent limitations when attempting to simulate high temperatures. Resistive elements emit in the infra-red because they operate at or just above the apparent temperature. Therefore, to simulate a $3000 \mathrm{~K}$ target, the resistive element must be at least this temperature. This places significant constraints on the materials and thermal management of the overall system. In addition, the peak in the black body spectrum of the resistive element does not overlap the detector spectral range leading to a large amount of lost power outside the detector band. Solomon and Bryant ${ }^{1}$, in a review of the physics behind resistive element arrays, found that resistive element arrays will require substantial materials research to satisfy the requirements of next-generation simulators. The new materials must be stable at the operating temperatures $(\sim 3000 \mathrm{~K})$ and compatible with thin-film processing techniques. In addition, the maximum pixel temperature becomes governed by radiative losses rather than input power, in direct contrast to the existing resistive elements. This changes the response time relative to standard arrays, increases the fall-time and limits the frame rate. Clearly a new approach to high temperature object simulation is required.

In order to meet the anticipated need for improved infrared point sources and arrays with high apparent temperature and short rise and fall times, a new class of light emitting diodes (LED) are being developed that are expected to mimic black

*j.lawler@atec-ahx.com phone 1301 699-1024; fax 1301 699-1028; www.atec-ahx.com

Technologies for Synthetic Environments: Hardware-in-the-Loop Testing XIII,

edited by Robert Lee Murrer Jr., Proc. of SPIE Vol. 6942, 69420E, (2008)

0277-786X/08/\$18 - doi: $10.1117 / 12.778719$

Proc. of SPIE Vol. 6942 69420E-1 
body sources with apparent temperatures as high as $3000 \mathrm{~K}$ with fast response times. The total power received by a detector is the integration of the product of the detector's spectral response and the sources' spectral radiant exitance over the detector spectral range. A black body at a given temperature emits a continuous radiation spectrum over a large range of frequencies, but it is only the overlap with the detector spectral range that generates detected power. As the temperature of the black body changes, so does the detected power. A monochromatic source, such as an LED, that emits in the detector's spectral region can simulate an apparent temperature by producing an equivalent detected power as the black body. In this manner, an LED can simulate a $3000 \mathrm{~K}$ black body temperature while the LED itself is thermally at a much lower temperature.

However, since the electro-optical efficiency of IR LEDs is rather low, a significant amount of waste heat is generated in an IR LED that is simulating such an elevated temperature. This waste heat must be removed with a minimum of thermal resistance in order to maintain the reliability and reproducibility of the LED device ${ }^{2}$. The efficiency of the electro-optical processes within the LED decrease with increasing temperature, so a temperature rise within the LED device may prevent an LED from producing sufficient IR radiation. Also, in arrays of IR LED elements localized heating can cause a non-uniformity of IR radiation across an array, since the electro-optical efficiency will then vary across the array. This paper reports on a study of the thermal behavior of IR LED arrays and suggests configurations that optimize the thermal performance of these devices.

\section{IR LED GEOMETRY}

One of the major aspects of the geometry of an LED array that affects its thermal management is the manner in which electrical connections are formed between the electro-photonic portion of the LED and the electronics that generates and controls the voltage and current used to drive the LED. Based on thermal management considerations, the preferred method is to "flip-chip" the LED array onto the array driver, with bond pads on the LED array lined up with a corresponding set of bond pads on the array driver. This arrangement, as shown in Figure 1, allows the heat that is generated in the active layer of the LED to be conducted through the bond pads and into the array driver, which can be mounted on a cold plate or on the cold finger of cryogenic chamber. The alternative configuration with electrical contacts mounted on the back side of the LED array substrate would require the heat to be conducted through two substrates before reaching the cold plate. The disadvantage of requiring the generated IR radiation to travel through the LED substrate can be mitigated by use of a transparent or nearly transparent substrate. Matveev et al. ${ }^{3}$ showed that this flip-chip configuration reduces the deleterious effects of thermal heating in the LED active layer while being operated at high currents.

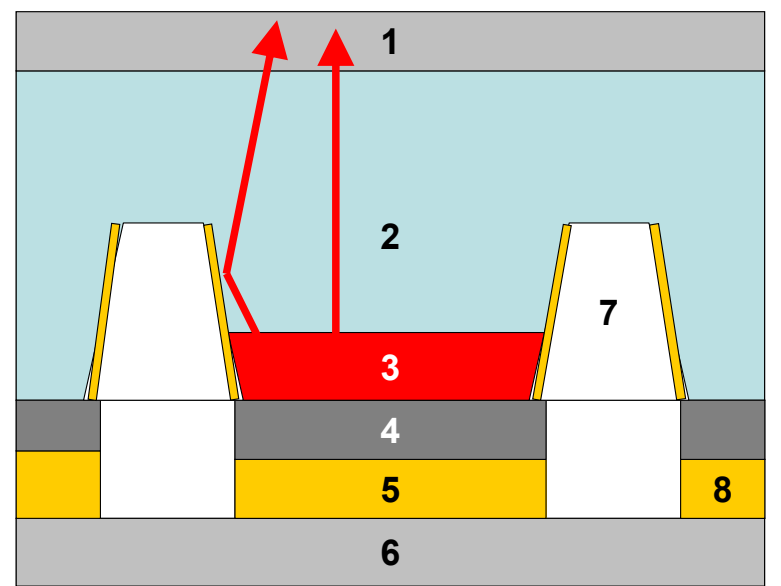

1. Outcoupling surface

2. Transparent substrate

3. Active layer

4. Soft metal contact

5. Bond pad (anode)

6. Array driver

7. Etch \& underfill

8. Bond pad (cathode)

Fig. 1. Schematic diagram of a flip-chip IR LED (not to scale) 


\section{THERMAL MODEL OF SINGLE LED PIXEL}

In order to quantify the thermal behavior of IR LED arrays, a model of a single LED pixel was developed using a commercial three-dimensional thermal analysis software package specifically tailored for analyzing electrical components (ICEPAK from ANSYS). A schematic diagram showing some of the components in the LED portion of the thermal model are shown in Figure 2. The red block represents the active LED layer, the gray blocks the etch surrounding the active layer, and the blue blocks are the bond pads for the LED pixel.
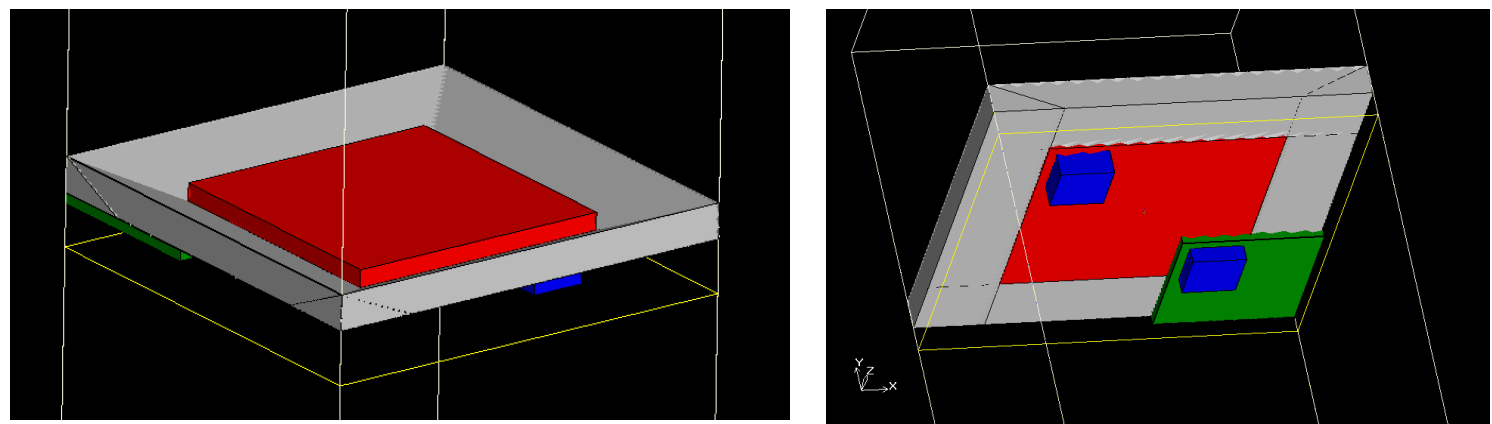

Fig. 2. Schematic diagram of some of the components in LED model

The array driver of the model consisted of alternating layers of metal and insulating (passivation) materials, with metallic vias embedded in the insulating layers providing electrical connections between the electrical layers. The design rules set forth by wafer fabrication foundries mandate that these vias must be small, restricted to a low number in any one area, and electrically relevant. That is, there is a limit to the size and number of the vias that can be included in these layouts to improve thermal transport across the insulating layers, and the addition of thermal vias that are not electrically active, which would improve heat transfer, would violate the standard wafer fabrication design rules. The metal layers in the driver arrays contain generally about 40 to $70 \%$ of metal traces, so the modeling was simplified by assuming all the metal layers in the array driver were solid metal layers. Several of the metal layers and the bond pads on the array driver are shown in Figure 3.

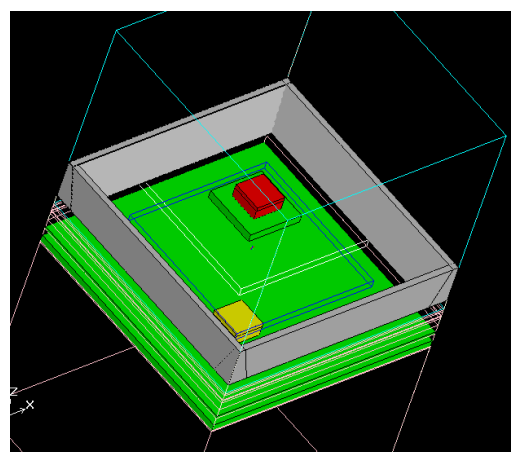

Fig. 3. Schematic diagram of some of the metal layers and bond pads in array driver

The thermal properties of all the materials in the model were based on literature values, including temperature-dependent thermal conductivities for most of the materials. As described below, the major thermal bottleneck is across the bond pads, since the volume in this region that is not part of the bond pads is filled with an "underfill" adhesive, which is an organic compound. For the simulations described below, the thermal properties of a commercially available underfill with a relatively high thermal conductivity $(0.7 \mathrm{~W} / \mathrm{m}-\mathrm{K})$ were used. The thermal conductivities of generic underfills are much lower - on the order of $0.1 \mathrm{~W} / \mathrm{m}-\mathrm{K}$ 


\subsection{Heat generation within active LED layer}

One of the issues in developing a thermal model of an IR LED pixel is specifying where in the LED the waste heat is being generated. To determine if the distribution of the generation of the waste heat within the active layer is an important parameter, a series of initial simulations were conducted in which the waste heat was generated in the entire active layer (cone shape), in a large portion of the active layer except for the center (donut shape), and in a somewhat narrow ring near the perimeter of the active layer (ring shape). Temperature contours for each of these configurations are shown in Figure 4. A summary of the maximum temperature rises in these three configurations for a series of active layers of varying diameters and waste heat loads is shown in Figure 5. The three different waste heat distributions geometries result in essential the same maximum temperatures. Therefore, the other LED models utilized for this study had the waste heat being uniformly generated throughout the entire active LED layer.
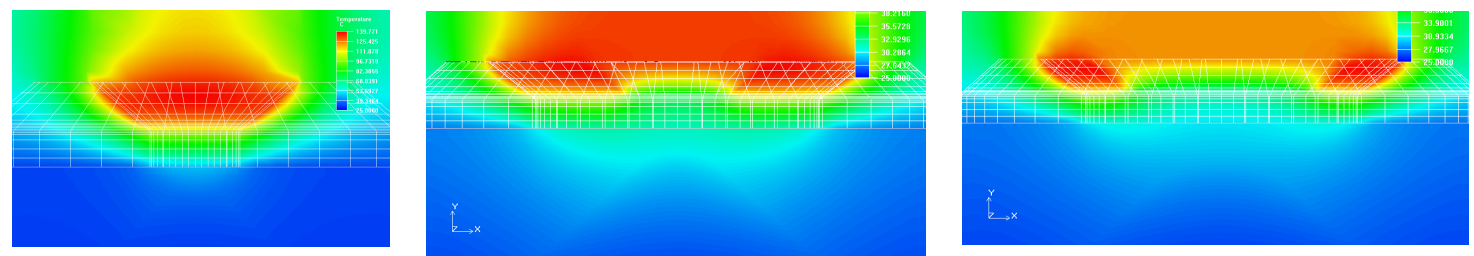

Fig. 4. Temperature contour plots of LED models with heat sources of different shapes (cone, donut, and ring) within the active layer.

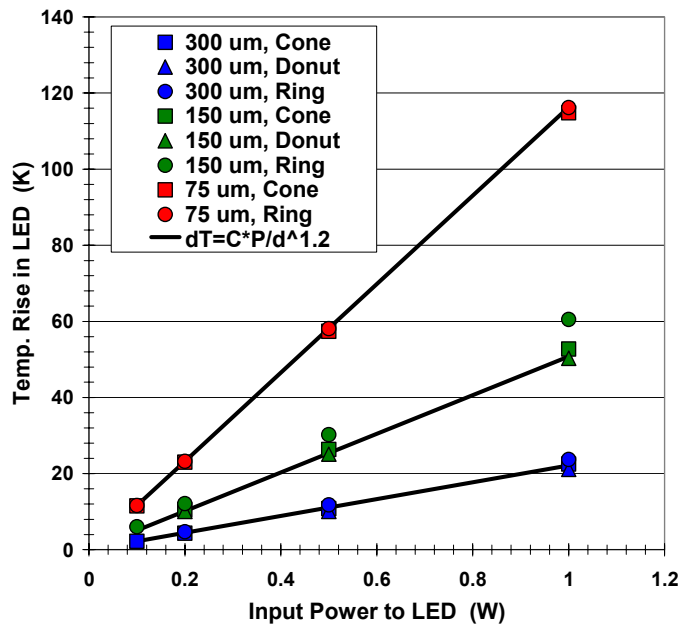

Fig. 5. Maximum temperature rises in LED thermal model with heat sourcesof different shapes for different power levels and LED diameters

\section{STEADY-STATE SIMULATIONS}

\subsection{Configurations with small bond pads}

A typical configuration for a flip-chip joining of two electrical components is for the bond pads on both components to be rather small, covering about 3-5\% of the unit cell for each LED pixel. These relatively small bond pads are certainly sufficient as electrical connections, but their small size creates a thermal bottleneck between the LED array and the array driver. With waste heat levels expected to be in the $50-200 \mathrm{~mW}$ range per LED pixel for generating the higher apparent temperatures the steady-state temperature rise in an LED can be greater than $100 \mathrm{~K}$ in geometries with small bond pads. The temperature rise is the difference between a local temperature within the LED and the temperature of the cooled side of the array driver's substrate. This level of temperature elevation may reduce the reliability of these IR LED arrays, reduce their maximum apparent temperature, and make the scenes generated by these arrays less accurate. 
Steady-state temperature contours within a single IR LED pixel with a $120 \mu \mathrm{m}$ pitch, being operated with a waste heat generation level of $0.2 \mathrm{~W}$, are shown in Figure 6 . The regions near the bond pads are relatively cool, but the relative temperatures of the portions of the active layer further from the bond pads become quite hot. The entire LED portion of the geometry is at an elevated temperature, and significant temperature gradients exist across the LED. Temperature profiles along the wafer thickness direction are shown in Figure 7. One profile cuts through the center of one of the bond pads, and the other profile runs through the center of the LED. The thermal transport through the underfill is so poor that most of the heat in this region moves laterally, which leads to the inflection point or cusp in the temperature profile. Temperature profiles across the active layer in an LED at different waste heat loads are shown in Figure 8 . A temperature variation of $40 \mathrm{~K}$ is generated in the active layer in an LED with a waste heat load of $0.2 \mathrm{~W}$.
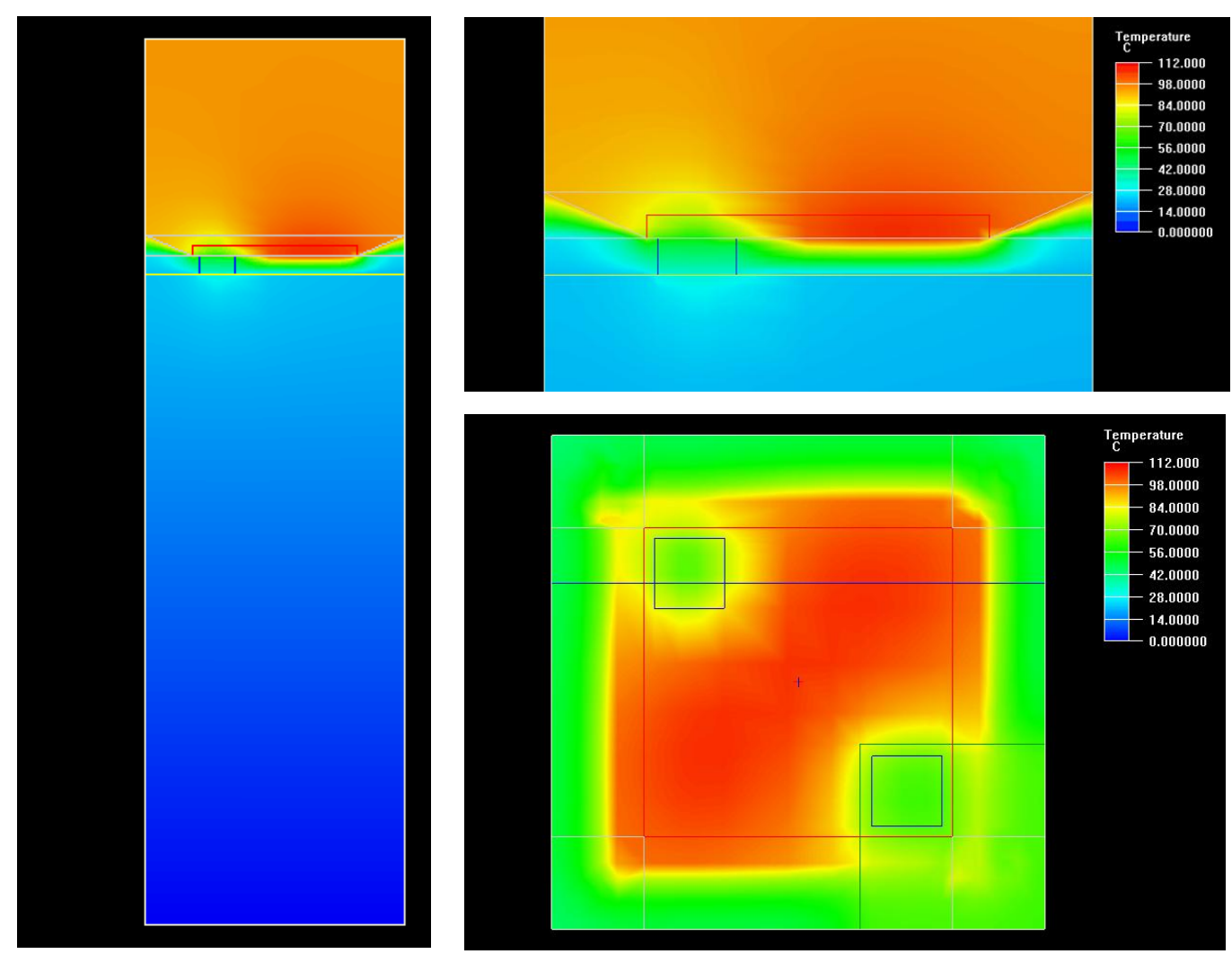

Fig. 6. Steady-state temperature contours in single IR LED pixel generating waste heat of $0.2 \mathrm{~W}$ 


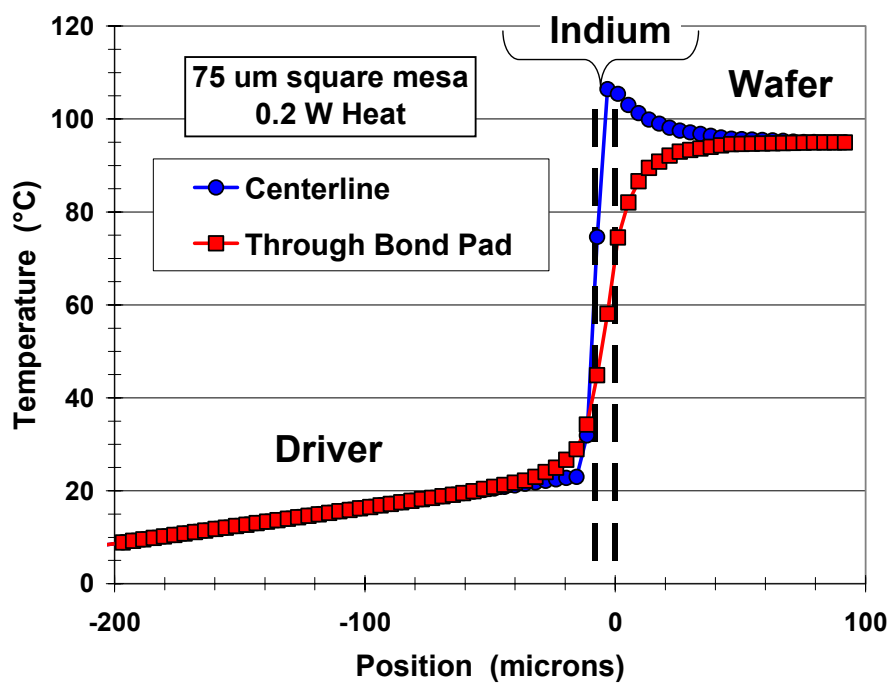

Fig. 7. Temperature profiles across LED array and array driverfor LED with small bond pads

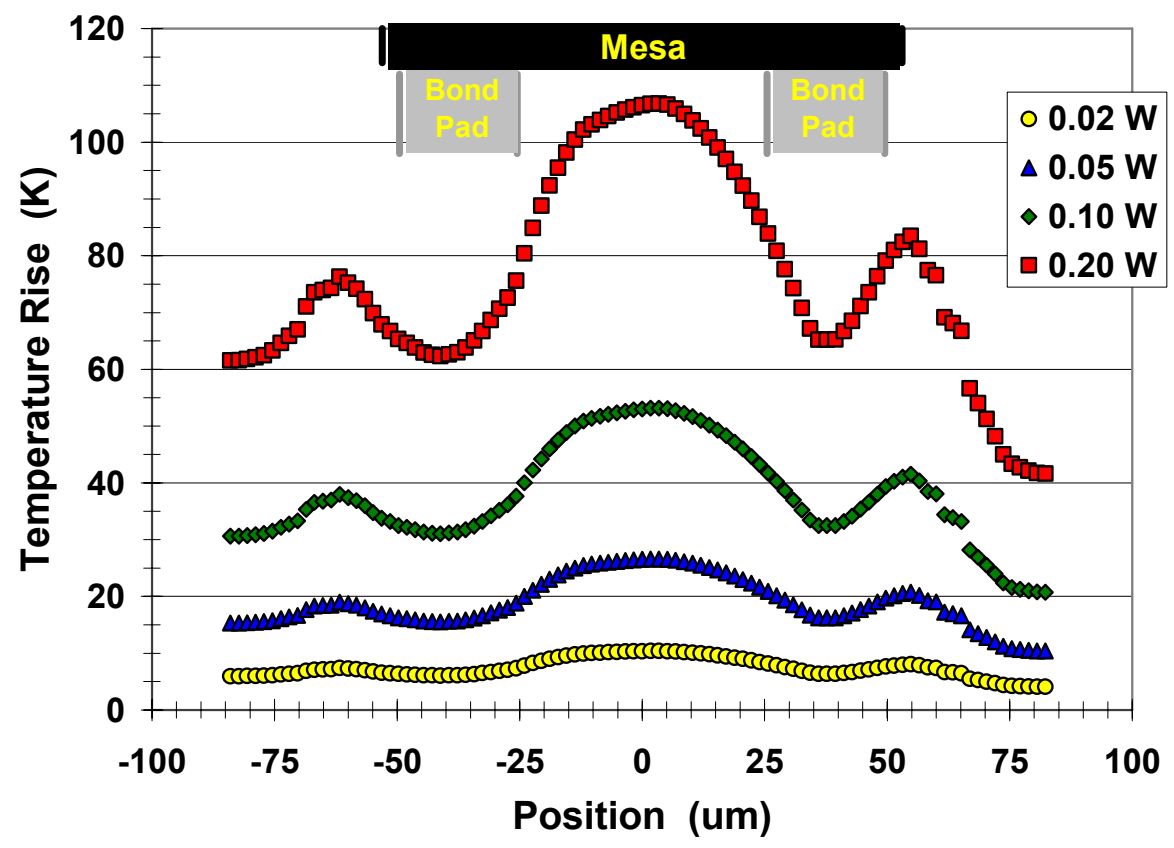

Fig. 8. Temperature profiles diagonally across active layer in LED array for LED with small bond pads

\subsection{Configurations with large bond pads}

One way to avoid the thermal bottleneck across the underfill is to increase the size of the bond pads. In order to demonstrate the effect of larger bond pads, the configuration of the bond pads was changed to one having a large central anode bond pad almost as large as the active layer and a rail cathode that surrounds the anode, as illustrated in Figure 9. A comparison of the resulting temperature contours is given in Figure 10. The temperature rise is reduced by a factor of 10 , and the temperature is quite uniform throughout the active layer. The temperature profiles across the active layer are 
shown in Figure 11 for these two configurations, as well as a third that incorporation of an outer rail in addition to the pair of small bond pads.

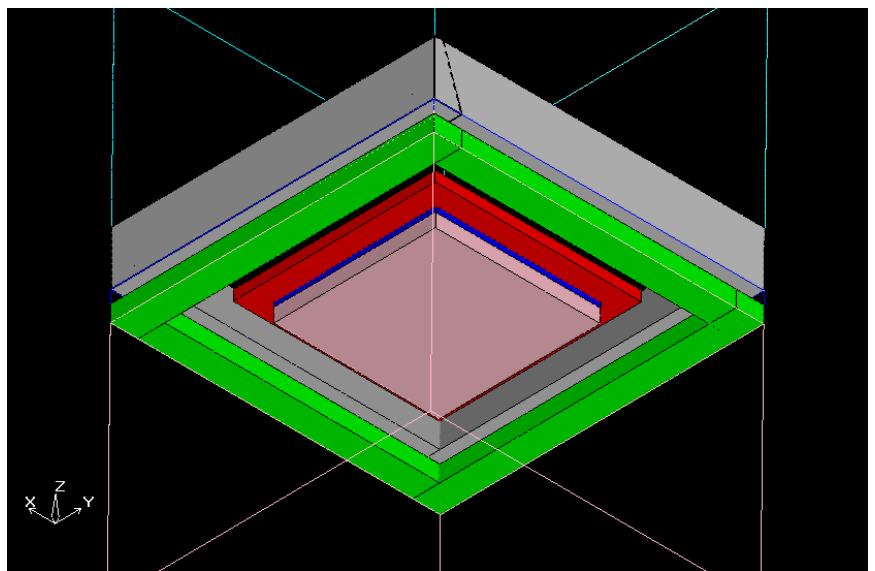

Fig. 9. Schematic diagram of LED with large central anode bond pad and surrounding rail cathode bond pad

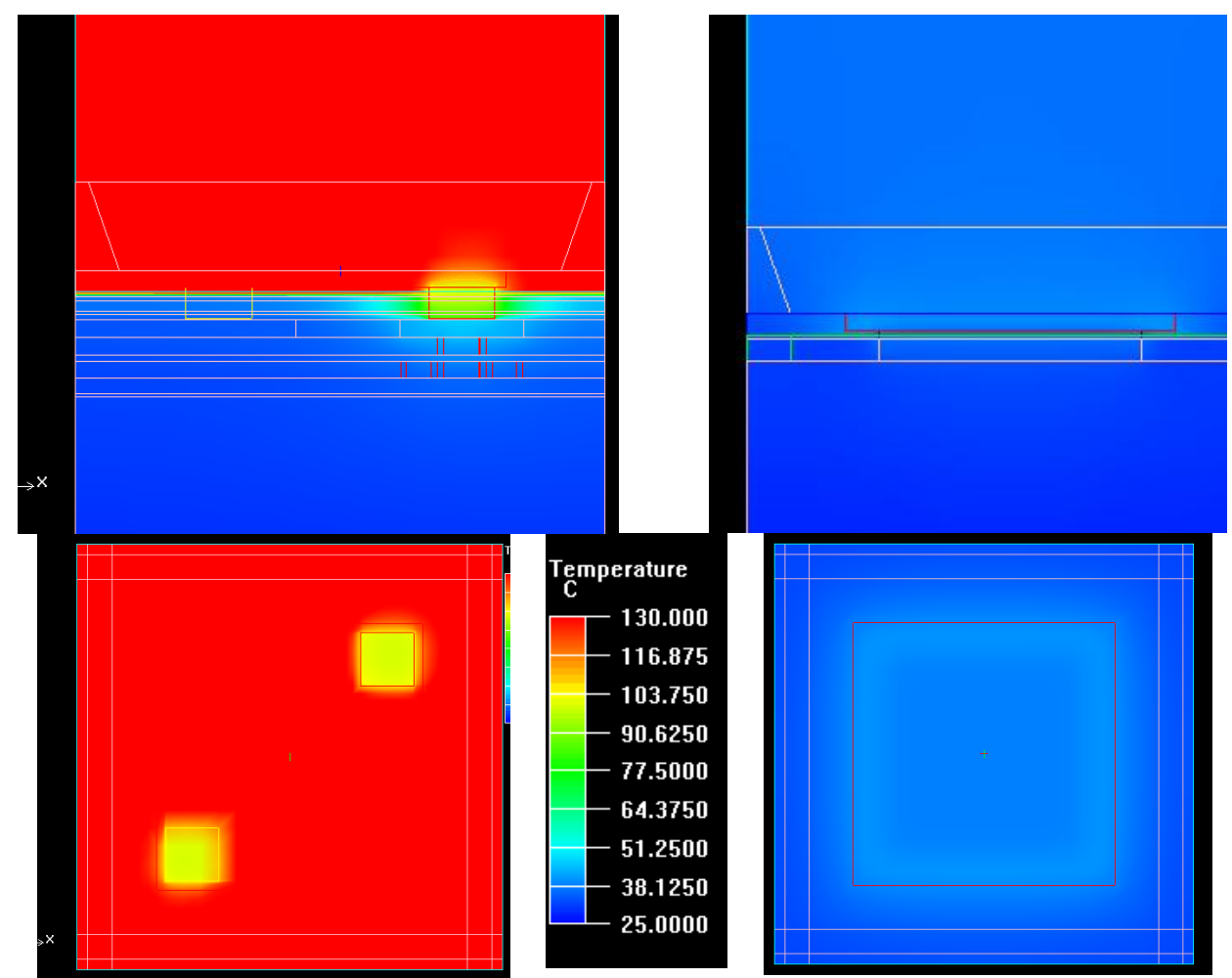

Fig. 10. Temperature contours in LED devices with small bond pads (left)and large central anode and rail cathode (right) 


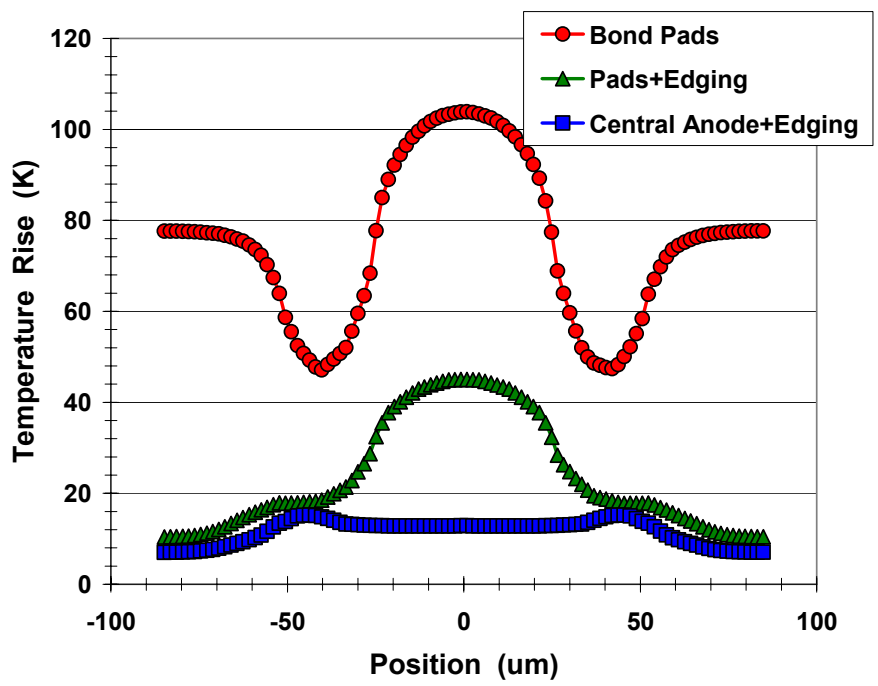

Fig. 11. Temperature profiles across active layer in LED array for LED with different bond pads

One issue with the use of a relatively large central anode and a long cathode rail bond pad is how these geometries will work with the standard bump bond deposition and flip-chip processing equipment and procedures. The use of small bond pads leads to uniform bump bond heights and lateral stability, so in order to conform to the standard processing, a more practical configuration may be multiple small bond pads that cover about the same area as the bond pads shown in Figure 9.

\subsection{Small array simulations}

The steady-state simulations of single IR LEDs presented above overstate the temperature rise in these devices for scene generation applications. In scene generation, only a portion of the array will be powered at any one time. The unpowered devices that surround a powered region (or the low powered regions that surround a highly powered region) will serve as additional thermal pathways for the waste heat. Simulations of a small 3x3 LED array were used to quantify the effect of unpowered neighboring LED on the maximum temperature rise in an IR LED. Symmetry boundary conditions were utilized in these simulations, so the $3 \times 3$ array actually represents a $6 \times 6$ array of LEDs.

The temperature contours from a simulation with a $2 \times 2$ sub-array powered at $0.2 \mathrm{~W}$ per LED are shown in Figure 12 . These contours show that heat is spreading into the surrounding unpowered LEDs. Temperature profiles across the active layer in an array with only one of the corner LEDs powered (representing powered 2x2 sub-array in a 6x6 array) are plotted in Figure 13. If the powered sub-array is this small, its many unpowered neighbors greatly reduce the maximum temperature. 

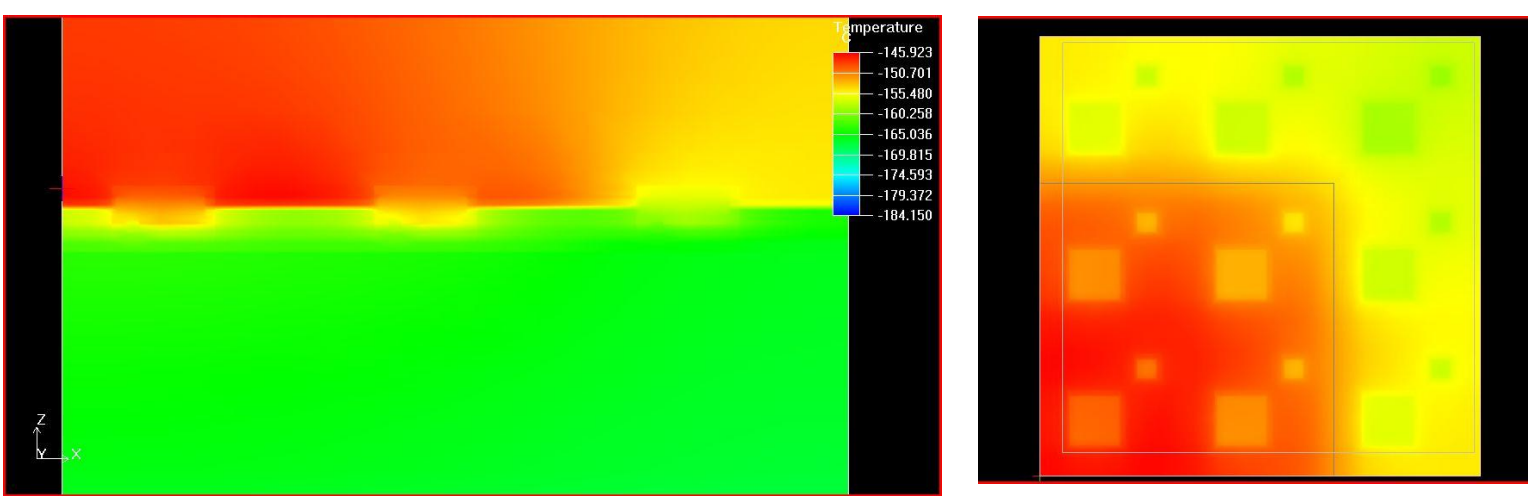

Fig. 12. Temperature contours in $3 \times 3$ LED array with power supplied to $2 \times 2$ sub-array $(0.2 \mathrm{~W} / \mathrm{LED})$

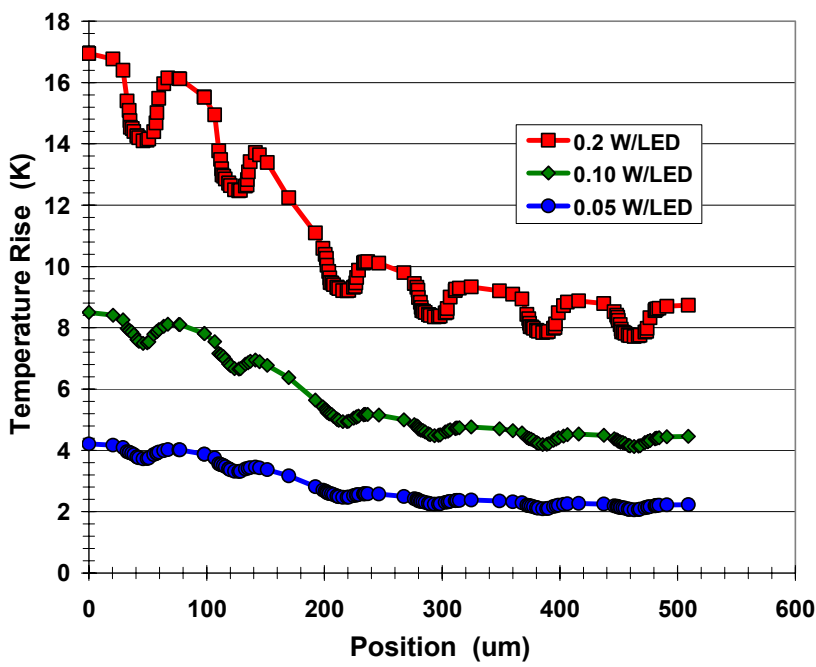

Fig. 13. Temperature profiles across active layer in 3x3 LED array with one corner LED powered at different power levels.

The maximum temperatures from simulations of varying powered sub-array sizes at different power levels are compared in Figure 14. The 6x6 "spot" (from powering all LEDs in 3x3 array) is equivalent to the single LED model presented above. There are no unpowered LEDs into which the heat can spread. The maximum heat in a $4 x 4$ spot is reduced by half due to the lateral spreading of the heat. A smaller $2 \times 2$ spot has a maximum temperature that is reduced by half again. However, these reductions in maximum temperature are for very small spots. The temperature in the middle of a $4 \times 4$ spot will be quite close to that of the fully-powered array, which is represented by the single LED array results presented above. Two simulations with larger bond pads (4x area of small bond pads) are included in this figure, which show that the combination of larger bond pads and powering only a sub-array are effective in combination to reduce the temperatures in LED arrays. 


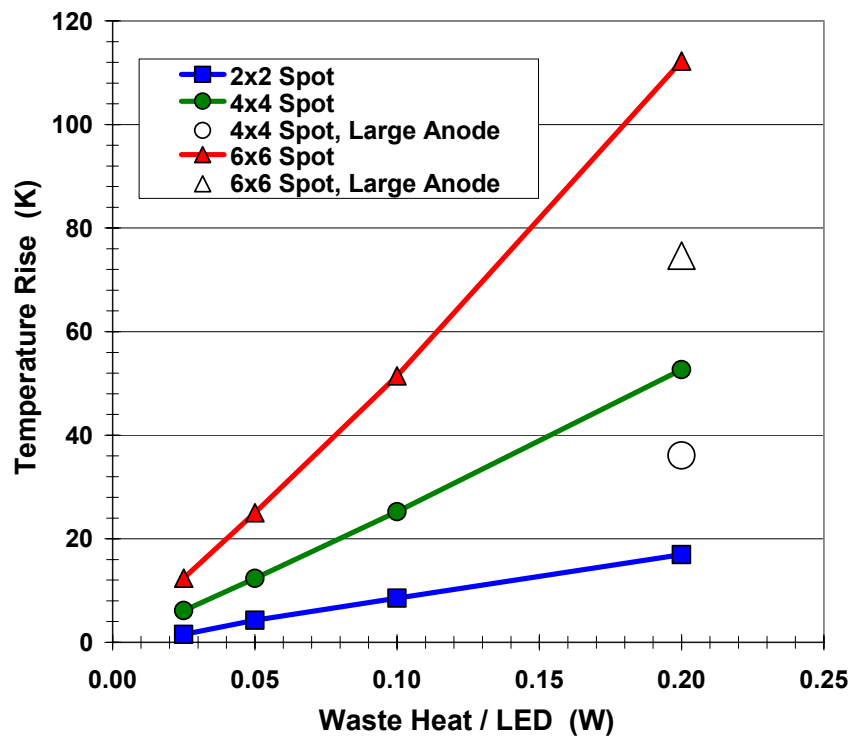

Fig. 14. Maximum temperatures as function of powered array size and power per LED. Results with $4 \mathrm{x}$ larger bond pad area are represented by unfilled symbols.

\section{TRANSIENT SIMULATIONS OF IR LEDS}

Besides being operating in a manner in which only some of the LEDs in the array are being powered at any one time, the scene generation application also has the characteristic that the scene will change with time. Thus, the transient characteristics of the thermal behavior of the LED arrays are relevant. Excess heating that lingers will alter the powercurrent behavior of the affected LEDs, which will modify the Non-Uniformity Correction (NUC) for the array. Also, regions at elevated temperature will increase the thermal background of the array, which reduces its signal-to-noise ratio.

Therefore, to characterize the temporal response of an LED array, a series of transient simulations were conducted. An illustrative example is shown in Figure 15. The rather elevated temperatures in the steady-state temperature contour have largely dissipated $1 \mathrm{~ms}$ after power was shut off to these LEDs, and the temperature gradients across the active layer have largely dissipated. After $3 \mathrm{~ms}$, almost no variation in temperature exists in the small array.

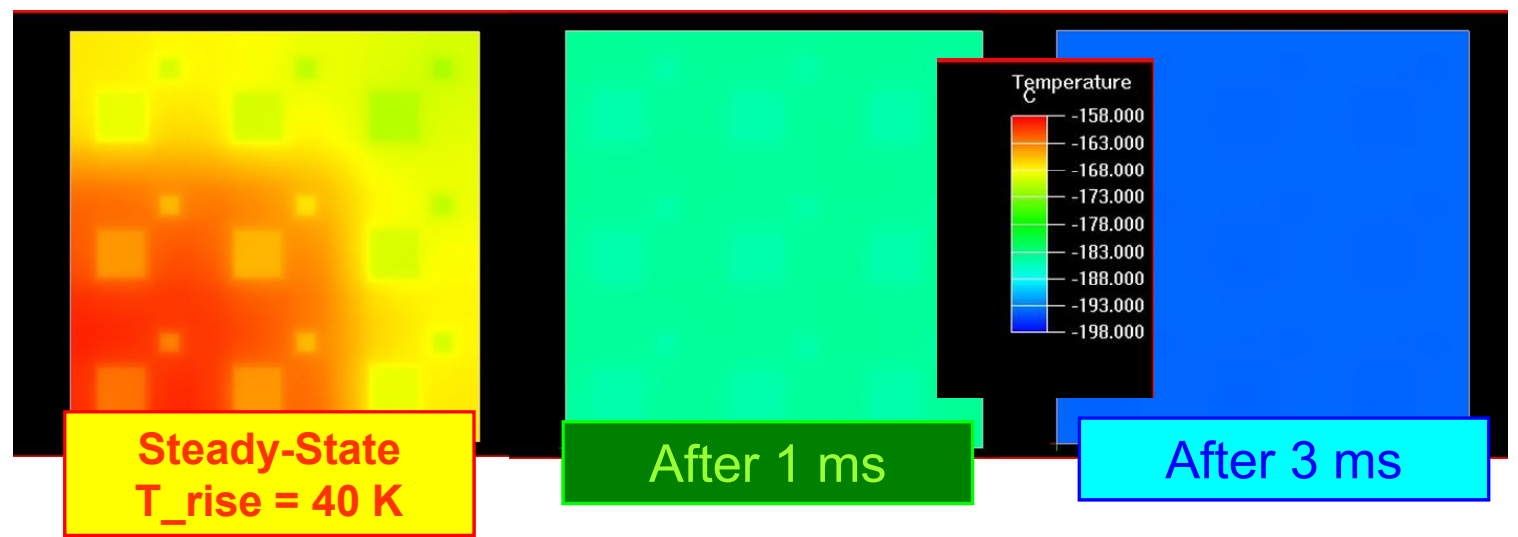

Fig. 15. Temperature contours in small $3 \times 3$ IR array at steady-state (left), $1 \mathrm{~ms}$ after turn-off (middle), and after $3 \mathrm{~ms}$ (right).

The temperatures in the active layers of two different LEDs (differing sizes of bond pads) are shown in Figure 16 as waste heat is applied to the active layers. The temperature rise data were fit to a function of the form: 


$$
T=T_{\max }\left(1-e^{-k t}\right),
$$

where $\mathrm{T}$ is the temperature, $\mathrm{Tmax}$ is the steady-state temperature, $\mathrm{k}$ is a time constant, and $\mathrm{t}$ is time. The same time constant was found to fit the data from both LEDs quite well. The temperature drop was fit to a related function:

$$
T=T_{\max } e^{-k\left(t-t_{0}\right)},
$$

where $t_{0}$ is the time at which the waste heat no longer being generated. This function fit the decreasing temperature quite well, as shown by the semi-log plot in Figure 17.

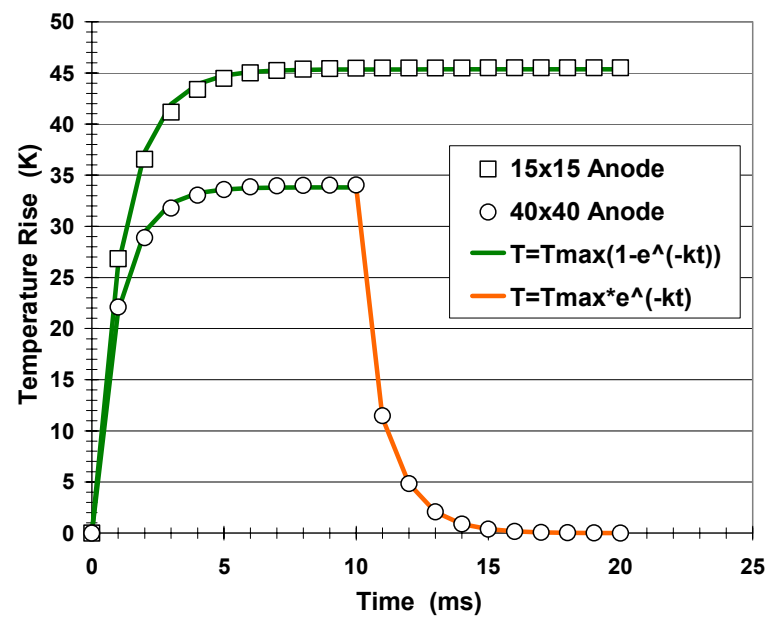

Fig. 16. Temperatures in active layers of two LEDs during cycling of power.

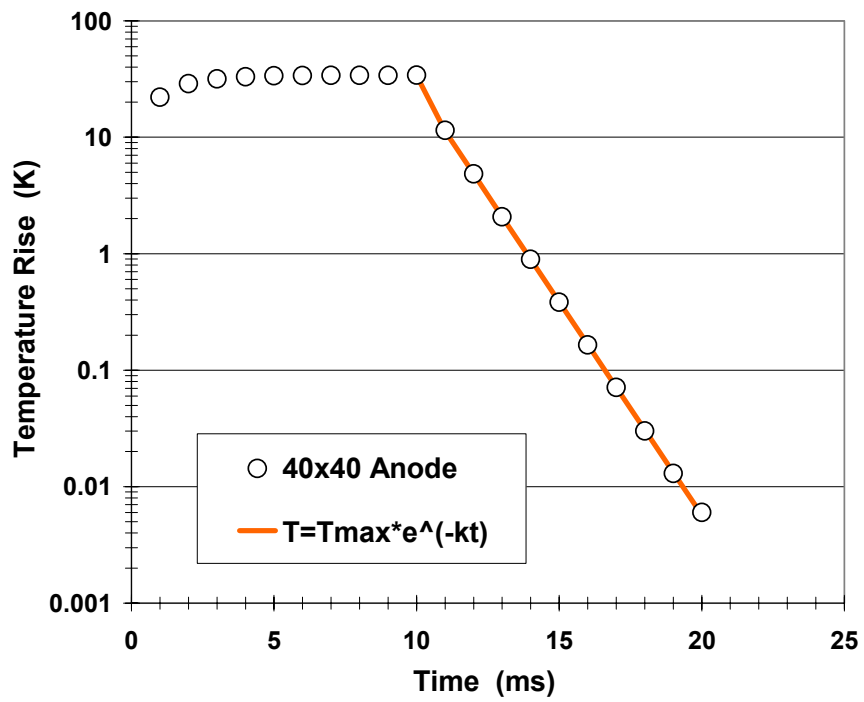

Fig. 17. Decreasing temperature in active layer after cessation of heating

\subsection{Pulse simulations}

In many scene generation testing situations, the objects in the scene are moving and often quite rapidly. Many of the LEDs being powered may not be powered long enough to reach a thermal steady-state. To show the effect of limited time at high power, a $100 \mu$ s pulsing was simulated in our single LED model. The temperature in the active layer as a function of time is shown in Figure 18. Although the steady-state temperature rise is about $45 \mathrm{~K}$, the maximum 
temperature during a pulse of this length is only $6 \mathrm{~K}$. To fit the rising and falling temperatures in this simulation, two time constants were required. The individual functions are shown in Figure 18 and listed in Table 1. Also in Table 1, are the calculated rise and fall times, with the rise time defined as the time required for the rising function to change from 0 to $90 \%$ of its asymptotic value and the fall time defined as the time required for the falling function to change from $100 \%$ to $10 \%$ of its initial value.

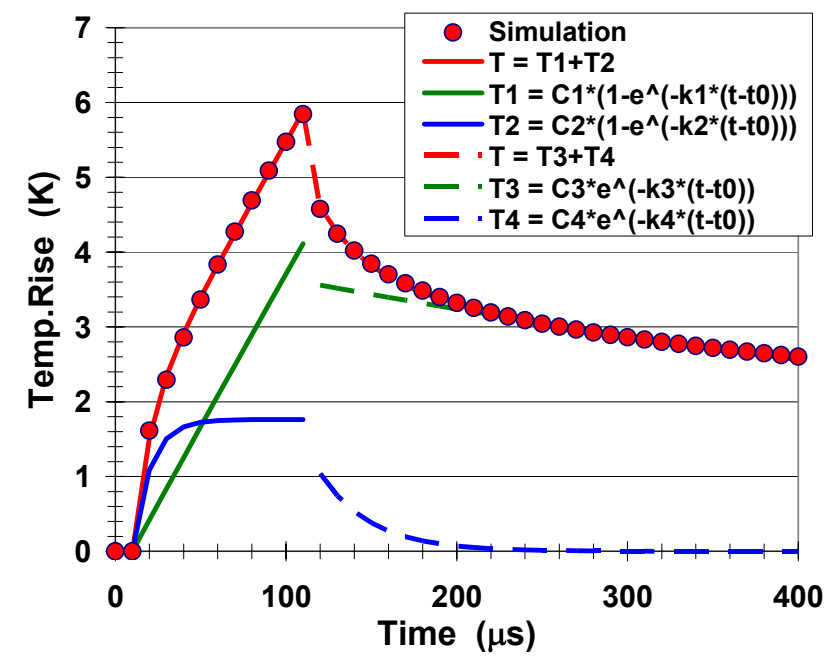

Fig. 18. Temperature rise during $100 \mu$ s powering of IR LED and subsequent cooling.

Table 1. Thermal time constants and rise and fall times for IR LED

\begin{tabular}{|c|c|c|c|c|}
\hline & $\begin{array}{c}\text { Heating Time } \\
\text { Constants }\left(\mathrm{s}^{-1}\right)\end{array}$ & $\begin{array}{c}\text { Rise Time (ms) } \\
\mathbf{0}-90 \%\end{array}$ & $\begin{array}{c}\text { Cooling Time } \\
\text { Constants }\left(\mathrm{s}^{-1}\right)\end{array}$ & $\begin{array}{c}\text { Fall Time (ms) } \\
100-10 \%\end{array}$ \\
\hline $\mathrm{A}$ & 410 & $\mathbf{5 . 7}$ & $\mathbf{1 2 0 0}$ & $\mathbf{2 . 0}$ \\
\hline $\mathrm{B}$ & $\mathbf{9 6 0 0 0}$ & $\mathbf{0 . 0 2 4}$ & $\mathbf{3 4 0 0 0}$ & $\mathbf{0 . 0 6 9}$ \\
\hline
\end{tabular}

Typically, the specified rise and fall times for a resistor array for scene generation are about $8 \mathrm{~ms}$. However, a fall time of $2 \mathrm{~ms}$ means that LEDs powered to high levels will remain at elevated temperatures for a significant period of time. These warmer sections of the array will not have the same electro-optical response as the cooler sections that have been dormant or at lower power. Based on further simulations of LEDs with different configurations, it was determined that the fall times can be reduced either by increasing the bond pad area or by reducing the size of the individual LEDs in the array. One example is shown in Figure 19. In this simulation, the LED pitch was reduced from 120 to $49 \mu \mathrm{m}$, and a large central anode with rail cathodes were substituted for the two square bond pads. The rise time in this simulation was about $1.1 \mathrm{~ms}$, but the fall time decreased to about $0.1 \mathrm{~ms}$. This decreased fall time should help eliminate the effects of previous power levels on the electro-optical responses of an IR LED and its neighbors in an IR LED array. If relatively small bond pads are used with the $49 \mu \mathrm{m}$ LED pitch, the fall time was found to be $0.48 \mathrm{~ms}$, so aspects of the geometry must be considered to reduce thermal effects. 


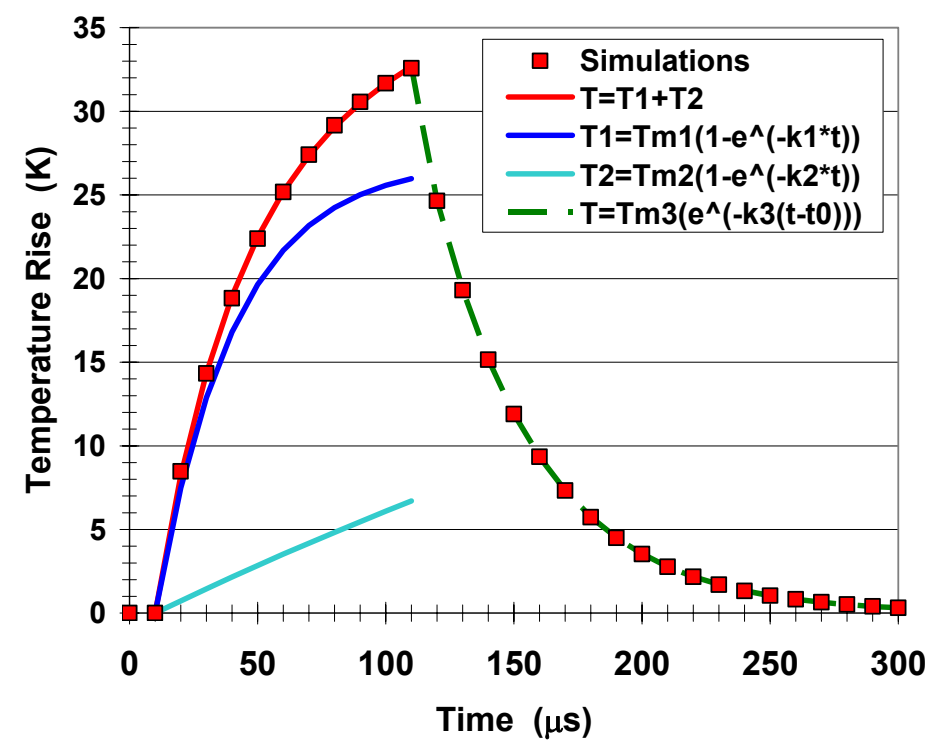

\section{CONCLUSIONS}

The temperature rise across IR LEDs can be significant if thermal management issues are not addressed during the design or layout of the LED and how the LED array is interfaced with its driver array. Significant amounts of heat must be transported across the bond pads of the LED, so the area covered by these bond pads should be a significant portion of the cross-sectional area of the interface between an LED pixel and its corresponding driver array element. Thermal simulations can be used to evaluate LED array configurations, including quantifying its thermal time constants and the effects of powered and unpowered LEDs on the thermal environment of their neighboring LEDs.

\section{ACKNOWLEDGEMENTS}

The authors would like to thank the Test Resource Management Center (TRMC) Test and Evaluation/Science and Technology (T\&E/S\&T) Program, Dr. Don Snyder, our MDA SBIR technical monitor, and Dr. Mark Massie and Nova Sensors for their support. This work is funded by the T\&E/S\&T Program as a sub-contract to the University of Iowa contract number W91ZLK-06-C-0006, MDA SBIR contract number W9113M-06-C-0166, and a contract with Nova Sensors.

\section{REFERENCES}

${ }^{[1]}$ S. Solomon and P. Bryant, 2003, “Adventures in high-temperature resistive emitter physics," SPIE Proc. 5092, 2003, 52-60.

[2] O. Kückmann, "High power LED arrays: Special requirements on packaging technology," Light-Emitting Diodes: Research, Manufacturing, and Applications X, Steubel, P., Yao, H.W., and Schubert, E.F. (editors), Proc. SPIE, Vol. 6134, 613404, (2006).

[3] Matveev, B.A., Zotova, N.V., Karandashev, S.A., Remennyi, M.A., Il'inskaya, N., Stus', N.M., Shustov, V., Talalakin, G.N., and Malinen, J., "InAsSbP/InAs LEDs of the 3.3-5.5 $\mu \mathrm{m}$ spectral range," IEE Proc.-Optoelectron., 145 (5) 254-256 (2002). 